Nicola Cirillo ORCID iD: 0000-0003-1429-1323

\title{
The molecular markers of cancer stem cells in head and neck tumours
}

\author{
By
}

Si Si Yu, Nicola Cirillo

Melbourne Dental School, The University of Melbourne, 720 Swanston street, 3053

Melbourne, VIC, Australia

*Address for Correspondence:

Prof. Dr. Nicola Cirillo

Melbourne Dental School

The University of Melbourne

720 Swanston Street, Carlton

3053 Victoria, AUS

Tel/fax. +610393411473

e-mail: nicola.cirillo@unimelb.edu.au

Data availability statement: Research data are not shared (N/A)

Running Title: head and neck cancer stem cell markers

\begin{abstract}
Head and neck cancer (HNC) is the six most common malignancy worldwide leading to more than 350,000 deaths annually. Despite recent advances in treatment modalities for these patients, there has been only a slight improvement of prognosis. As cancer stem cells (CSCs) have been implicated in tumour cell survival, progression, and response to therapy, the identification of this tumour subpopulation would have important therapeutic and prognostic implications. In this structured appraisal of the literature, Embase, PubMed and Ovid were searched for publications that investigated CSC markers of HNC in humans. The search was conducted under This is the author manuscript accepted for publication and undergone full peer review but has not been through the copyediting, typesetting, pagination and proofreading process, which may lead to differences between this version and the Version of Record. Please cite this article as doi: 10.1002/jcp.28963.
\end{abstract}

This article is protected by copyright. All rights reserved. 
the PRISMA guidelines with clear inclusion and exclusion criteria for articles published in the last two decades. The review process resulted in the identification of some key CSC markers such as CD44, ALDH1, CD133, Oct3/4, Nanog and Sox2, although a single common CSC sorting marker could not be found. These biomarkers were identified in a range of HNCs but the most common one was squamous cell carcinoma (SCC), predominantly oral SCC. Patient cohorts were of variable size (3 to 195 individuals) and the most common technique used for detection was immunohistochemistry. Some of the molecules were associated with poor prognosis and may be able to inform the choice of an appropriate treatment for these patients.

\section{Graphical Abstract}

This review summarises current evidence supporting the use of biomarkers for sorting cancer stem cells in head and neck tumours. Some of these molecules are associated with poor prognosis and may be able to inform the choice of an appropriate treatment for these patients.
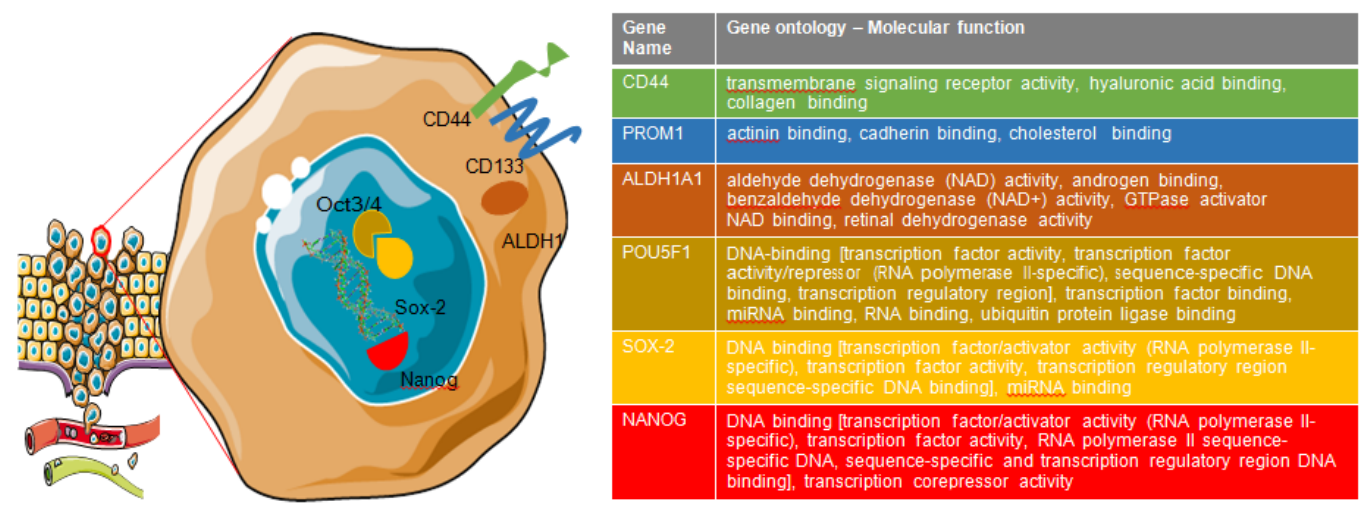

Keywords: Cancer stem cells, head and neck cancer, oral squamous cell carcinoma, CD44, CD133

\section{Introduction}

Head and neck cancer (HNC) includes a wide range of tumours encompassing structures such as the oral cavity, paranasal sinuses, nasal cavity, pharynx and larynx. (Argiris, Karamouzis, Raben \& Ferris, 2008) Importantly, HNC is the sixth most common malignancy worldwide with approximately 630,000 new cases diagnosed annually leading to over 350,000 deaths every year. (Parkin, Bray, Ferlay \& Pisani, 2005) More than $90 \%$ of HNCs are squamous cell carcinomas (HNSCC) that arise 
from mucosal surfaces of the oral cavity, oropharynx and larynx. (Vigneswaran \& Williams, 2014)

There are a multitude of risk factors for the development of HNSCC that include genetic background, diet, habits, geographic and socioeconomic factors. Amongst the habits adopted by patients, cigarette smoking and excessive alcohol consumption have a synergist effect and are the most important risk factors for developing HNSCC. (Morse et al., 2007) Furthermore, malignancies in the head and neck area, particularly the most common types (HNSCC, OSCC) are associated with high morbidity and high fatality. Despite recent developments in treatment options, the disease-free and overall survival of HNC patients has barely improved over the last decades. (Murdoch, 2007) This is partly due to resistance to treatment, which is thought to be driven by specific populations of cancer stem cells (CSCs).

One of the recent advances in the innovative treatment modalities of malignancy involves targeting CSCs, which possess intrinsic biological properties normally associated with stem cells and are able to reconstitute a tumour that is identical to the parental tumour. (Allegra et al., 2014) The cancer stem cell hypothesis proposes that CSCs are the original cells constituting the tumour, and these subsets of cells are responsible for tumorigenesis, tumour differentiation, tumour maintenance, spread and collapse. (Clarke et al., 2006) The pool of CSCs rarely divide and remain undifferentiated to guarantee the regeneration of new CSC. (Hur, 2009) The presence of a subpopulation of CSCs with self-renewal and multipotency properties has potential impact on the prognostic and therapeutic consequences of head and neck tumours. Therefore, when CSC populations are eliminated or targeted, it is more likely that the treatment will be effective, and relapse will be less likely. (Hur, 2009) Given that CSCs are thought to be resistant to, or be able to escape, conventional treatment, developing an effective strategy to target these cells is imperative if we are to improve cancer treatment and prognosis. The main target therapy approaches against CSCs involve improving sensitisation of CSCs to conventional drugs, promoting CSC differentiation, targeting and blocking relevant CSC signalling pathway and destroying CSC niches. (Garvalov \& Acker, 2010) Thus, the identification of biomarkers that are associated with CSCs is key.

This article is protected by copyright. All rights reserved. 
In the head and neck region, the identification of reliable and specific CSC markers for HNSCC would be critically important for diagnosis, treatment, and prognosis of these tumors. Recently, several studies have tried to identify markers of CSCs that can be targeted for therapeutic purposes. In addition, several molecular biomarkers have been used in clinical trials to sort and define CSC. In this study, we aim is to systematically appraise the existing literature about molecular markers of CSCs in head and neck tumours. Furthermore, we assessed whether these markers had any association with patient prognosis.

\section{Methods}

\section{Protocol and Search Strategy}

The protocol for this structured review has been in accordance with the PRISMA guidelines (Liberati, 2009). This study was performed to identify the specific molecular markers that are common in head and neck tumours and have been consistently identified in the literature.

A search was conducted July to September of 2018 using the following databases: MEDLINE OVID, PUBMED and Embase. The databases were searched for publications based on the title, abstract, language and relevance. The key words used were Head and neck tumour, cancer stem cells and molecular markers, as well as their synonyms (Table I). Gene ontology (molecular function) was searched via Uniprot (www.uniprot.org) .

\section{Eligibility criteria}

Articles were included in this review with the following criteria: 1) Written in English language; 2) Studies based on humans or human tissues (both In vivo and In vitro; 3) Cohort studies; 4) Retrospective studies; 5) Prospective studies; 6) Patients with confirmed diagnosis of Head and Neck cancer; article written between 1980 and 2018. Articles were excluded in this systematic appraisal based on the following criteria: 1) unpublished articles or conference proceedings; 2) Case reports, case records and letter to the editor; 3) Studies done on non-human samples; 4) studies where patient's diagnosis is uncertain (e.g. no histopathological confirmation); 5) Abstracts (Supplementary Table I). 
Study selection, data collection and data items

The process of study selection was divided in two phases. In the first phase, one author (SSY) screened for articles by reading only title and abstract of the studies under the guidance of the senior author (NC). The full-text of publications meeting the initial inclusion criteria were analyzed in the second round by both authors. At the end of the second phase, the two reviewers provided independently a final judgement (include, exclude or uncertain) of inclusion for the selected articles. In cases of disagreement, the senior author (NC) took a final decision of the inclusion after discussion with the first reviewer in a joint meeting. At the end of the selection process, papers fulfilling all inclusion criteria were included in the critical review.

Data extraction was performed by SSY using an ad hoc extraction sheet; subsequently, data were double checked in a joint session. The following parameters were extracted from each included study, where available: name of the first author, year of publication, nation where the study was performed, head and neck tumor sub localization, CSC biomarker used, rate of metastasis, overall survival (OS), diseasefree survival (DFS) and progression-free survival (PFS).

\section{Results}

Study selection process and study features

The search of all databases mentioned above yielded 534 results, and 342 publications remained after duplicates were removed. The duplicates were removed via EndNote and cross referenced with manual screening. The articles were selected for the criteria of English language, human studies and full texts. After selection, 61 journal articles fitted the selection criteria. Of these publications, 31 remained after screening by abstracts. After reading the full text and critically analysing the articles, a further 18 studies were excluded from the data set. These studies were excluded because of relevance to the topic of Head and Neck Tumours and CSCs. Furthermore, some of these studies did not have histopathological confirmation of the patient's with Head and Neck Cancer. The remaining 13 studies were eligible for detailed assessment for 
this systematic review. Details of the selection process are provided in Figure 1. Overview of study characteristics of articles that were eligible for analysis is reported in Table II.

Although articles were selected from 1998, it can be seen that the articles that were eligible for this review are from 2010. The publication years demonstrate that the detection of molecular markers of head and neck cancer stem cells has been a relatively new field that has emerged over the last decade. Overall there have been a total of 15 molecules that have been identified as putative markers of head and neck cancer stem cells, some of which have prognostic significance (Table III).

The most common technique used amongst these studies to assess the biomarkers was immunohistochemistry (IHC). The results of cancer types were consistent with previous literature (Linge et al., 2016) where HNSCC, particularly those in the oropharynx and larynx region, were the most common (10 studies out of 15, 67\%). The number of participants of the included studies ranged from 3 to 195 individuals and the median was 50 participants. Below are reported the six most researched markers (Figure 2).

\section{CD44 as a CSC marker}

CD44 is the major receptor for hyaluronan and constitutes a single chain transmembrane glycoprotein that is widely expressed in physiological and pathological conditions (Destro Rodrigues et al., 2016) This molecular marker has been identified in six out of the thirteen studies included in this review. In general, CD44 is a widely explored CSC marker when it comes to HNSCC. (Faber, 2011) Studies show that CD44 levels can predict the local recurrence after radiotherapy in patients with early-stage laryngeal cancers. (de Jong et al., 2010) Linge et al found that CD44 expression was significantly associated with the primary endpoint locoregional tumour control. This study also concluded that patients with surgically resected tumours with no detectable CD44 protein expression have increased locoregional tumour control rates compared with those with CD44 protein-expressing tumours. (Linge et al., 2016). Rodrigues et al showed CD44 was expressed in normal glandular tissue as well as neoplastic tissue, and in all cases of Mucopidermoid Carcinoma (MEC). Furthermore, in some cases, CD44 expression was higher in the 
tumour invasive front than in the central parts, indicating that CD44 may participate in MEC progression. (Destro Rodrigues et al., 2016) In some cases of HNSCC, expression of CD44 was associated with worse prognosis, poor differentiation and metastasis. (Soave et al., 2013). Seino et al investigated CD44 combined with higher ALHD1 enzymatic activity and demonstrated that these cells had a higher tumour sphere forming ability as well as greater tumorigenesis. (Seino et al., 2015). Mannelli et al gathered that the role of CD44 could contribute to epithelial-mesenchymal transition and cancer progression, as well as lymph node metastasis. CD44+ cells have a very high clonogenic capacity and are the main population proliferating in in vitro cultures. (Mannelli et al., 2015) Huang et al shows that CD44 is overexpressed in Tongue SCC, it has major roles in CSC self-renewal and differentiation, and that CD44 positive expression was mainly detected in the membrane and was strongly positive in 26 out of 66 specimens and weakly positive in 17 out of 66 specimens..

\section{ALDH1 as a CSC marker}

ALDHA1/ALDH1 is a human aldehyde dehydrogenase family and has been shown to be useful for identification of normal stem cells and CSCs (Ma \& Allan, 2010). ALDH1 has also been a common recurring CSC marker identified in this review present in 4 out of the 13 articles reported.

In their study, Kim et al associated the expression of ALDH1 with metastasis; specifically, patients who expressed ALDH1 developed metastasis more frequently (statistically significant difference) than those who were negative for this marker. Thus, ALDH1 can be used as a predictive marker for metastasis particularly in those with eyelid sebaceous gland carcinoma. It would be a useful prognostic indicator and could facilitate the selection of patients who require more aggressive treatments to reduce the chance of metastasis. (Kim, Choung, Lee, Khwarg \& Kim, 2015).

ALDH1 has been found to mediate oxidation of Vitamin A precursor which plays important roles in cell growth, differentiation and tissue homeostasis. Seino et al concluded that ALDH1 is one of the key players in maintaining CSCs and indicated that its presence may be involved in HNSCC tumour invasion and metastasis. (Seino et al., 2015)

This article is protected by copyright. All rights reserved. 
Huang et al also associated ALDH1 with tumour malignancy and self-renewal properties of stem cells in head and neck carcinomas, where ALDH1 was expressed in the cytoplasm of cancer cells (its expression being weakly positive in 24/66 cases and strongly positive in 18/66 spicimens). Despite this, ALDH1 in this study was not significantly associated with the overall 5-year survival rate. Although ALDH1 was important in the maintenance of CSCs, this study did not correlate ALDH1 with lymph node metastasis, in contrast to previous studies. (Huang, $\mathrm{Xu}, \mathrm{Wu}, \mathrm{Sun} \&$ Zhang, 2014). Importantly, this research was one of the first to show associations of ALDH1 with other CSC markers such as Sox 2.

Yang et al was one of the first studies to reveal the association of ALDH1 with oesophageal SCC. Furthermore, ALDH1 positive cells were preferentially distributed in the invasion front of oesophageal SCC and metastatic lesions. In vitro and in vivo results from this research also demonstrated that cell lines with high ALDH1 levels were more highly invasive and metastatic. Michifuri et al showed for the first time that ALDH1 was associated with lymph node metastasis in OSCC cases.

From the analysis of current literature, we conclude that whilst ALDH1 does not have prognostic significance, this CSC marker could be used for prediction of lymph node metastasis (Michifuri et al., 2012) in site-specific HNCs.

\section{CD133 as a CSC marker}

Patients with CD133-positive tumours exhibited shorter metastasis-free survival compared with patients who did not express this marker. (Kim, Choung, Lee, Khwarg \& Kim, 2015) Although its role in HNC is still relatively unknown, CD133 has previously been strongly correlated with liver metastasis in colon cancer. High levels of CD133 might be a novel marker for predicting the poor prognosis of patients with oesophageal SCC and could have the potential to serve as therapeutic targets in these types of cancers (Lu et al., 2015). However, the study by Mannelli et al (2015) contradicted previous results by showing that CD133 expression was low in frequency in tumour specimens and in cell cultures. Despite this, they showed that the few cases positive for CD133 were significantly associated with cervical lymph node metastasis (Mannelli et al., 2015). The authors concluded that CD133 had a role in HNSCC progression. Perhaps the reason this study showed limited expression of 
CD133 was because the number of patients with metastasis in their cohort was small. Specifically, this study only examined 29 patients and only 3 cases were positive for CD133; however, all 3 were associated with metastasis. Similarly, Tang et al (Tang et al., 2013) could not find CD133 expression in any of the primary tumours examined, although their sample size was small (8 individuals). Thus, it is possible that CD133 is only present in a small subset of lymph node metastasis sites.

\section{Oct3/4 as a CSC marker}

Oct4 is a transcriptional factor that is important in maintaining the pluripotent and undifferentiated state of embryonic stem cells. (Kashyap et al., 2009) Previous studies in literature have associated Oct4 with tumour progression and poor prognosis. Rodrigues et al found that Oct4 expression was high in the nucleus and cytoplasm of tumour cells in 20 out of 28 cases. (Destro Rodrigues et al., 2016) Not only was Oct4 present in the majority of MEC cases but, also, was associated with perineural invasion which is one of the most significant prognostic factors for MEC. (Destro Rodrigues et al., 2016) However, in this study, the association between Oct4 and overall survival was not investigated. There was a strong link showing that CSC markers like Oct4 can enhance tumourigenic potential of malignant MEC cells (Destro Rodrigues et al., 2016). In other retrospective clinical studies, it was also found that Oct4 protein expression was associated with further disease progression, greater rates of metastasis, resistance to therapy and overall worse survival. (Chang et al., 2008)

The study by Habu et al (2015) showed that higher expression of both Oct4 and Oct3 increases the malignant potential and that when Oct3/4 was inactivated, this induced regression of the malignant component (Habu et al., 2015). It is proposed that the reason Oct3/4 promote the formation of metastatic lesions, when combined with other CSC markers such as Nanog, is due to their enhanced migration and invasion abilities. In addition, when malignant lesions were present with Oct3/4 and/or Nanog in primary cancer cells, this feature was a predictor for high risk patients of delayed neck metastasis. (Habu et al., 2015) Huang et al determined that the positive expression of Oct4 was mainly located in the nucleus and it was over expressed in tongue SCC in 35 of 66 specimens studied. Overall, the available literature suggest that Oct3/4 may 
promote a malignant/metastatic phenotype, particularly when co-expressed with other CSC markers.

\section{Nanog as a CSC marker}

Nanog is a variant homeobox transcription factor which is one of the primary downstream targets of Oct3/4. (Chambers et al., 2003) Nanog was studied with Oct4 in MEC in one study (Destro Rodrigues et al., 2016) and it was identified that Nanog, like Oct 4, was a CSC marker for MEC, a type of HNC. This study demonstrated that Nanog was highly expressed by neoplastic cells in perineural areas and similar to Oct 4 it was correlated with tumorigenesis, loss of differentiation, invasion and metastasis. (Destro Rodrigues et al., 2016)

Seino et al showed that HNSCC cells with high expression of Nanog were resistant to radiation and anti-cancer treatments. In this study the expression of Nanog was also associated with the expression of Sox1. (Destro Rodrigues et al., 2016)

Habu et al (Habu et al., 2015) identified high levels of Nanog expression present in OSCC CSCs, which is consistent with studies published previously. (Boiani \& Schöler, 2005) Furthermore, like its upstream targets Oct3/4, Nanog has been proposed to contribute to enhanced cell motility and invasiveness of HNSCC cells (Habu et al., 2015). Thus, the authors concluded that Nanog in primary cancers can be considered a predictor for stratifying patients at a high risk of distant neck metastasis, thus informing the treatment planning for these patients. Huang et al investigated the relationship between Nanog and OSCC and showed that Nanog-positive cells exhibited hallmark CSC properties (Bourguignon, Wong, Earle \& Chen, 2012). Specifically, these cells displayed properties of self-renewal/clonal formation and the ability to generate heterogeneous cell populations. (Huang, $\mathrm{Xu}, \mathrm{Wu}$, Sun \& Zhang, 2014)

Overall, these studies suggest that Nanog can serve as CSC marker and is predictive of poor prognosis.

Sox2 as a CSC marker

This article is protected by copyright. All rights reserved. 
The Sox2 gene encodes a transcription factor belonging to the Sox family that is critical for maintenance of self-renewal in stem cells and neural progenitor cells. (Adachi, Suemori, Yasuda, Nakatsuji \& Kawase, 2010) It was only recently discovered that Sox2 plays a role in malignancy stemness and as a possible prognostic factor. (Ivanova et al., 2006) Huang et al revealed that Sox2 protein was mainly located in the nucleus and was strongly positive in 20 out of 66 specimens and weakly positive in 21 out of 66 specimens.

Previous studies concluded that Sox2 is usually co-expressed with CD44 and both these factors in combination play a prominent role in self-renewal capacity of CSC. (Saito et al., 2011) However, in one study, Sox2 expression was not significantly associated with 5-year overall survival based on the Kaplan-Meier analysis. Despite this, the authors still suggested that Sox2 could play an important role in carcinogenesis and could possibly be used as a prognostic indicator for patients with OSCC in the future. Michifuri et al (Michifuri et al., 2012) showed that Sox2 expression as a CSC marker was associated with ALDH1 and there was a link between these two markers and OSCC metastasis. (Michifuri et al., 2012)

\section{Discussion}

Our review of the current literature shows that several CSC molecular markers can be identified in HNC and that some of these are predictive of a more aggressive biological behaviour and poor prognosis. The studies with the strongest evidence were those that had a large patient data base as well as those based on fresh patient tissue samples, these being Linge et al (Linge et al., 2016), Lu et al (Lu et al., 2015) and Yang et al (Yang et al., 2013). In contrast, there were some studies with very few samples, notably Seino et al (Seino et al., 2015), Tang et al (Tang et al., 2013) and Karatas et al (Karatas et al., 2016).

The literature search reported in this present study was conducted systematically; however, it should not be considered a systematic review strincto sensu according to the guidelines reported in the Cochrane Handbook and the Preferred Reporting Items for Systematic Reviews and Meta-Analyses (PRISMA) statement: research question, nature of the topic under investigation, and heterogeneity of the models and techniques used do not allow to fulfil the criteria of a systematic review of the 
literature. For example, the majority of studies examined were conducted retrospectively with fresh or frozen tissue samples, and some studies were conducted in vitro on cell lines (Seino et al., 2015). In the latter case, it is known that culture medium, chemicals and experimental techniques can all influence tumour cell growth as well as expression of markers. Some studies (Destro Rodrigues et al., 2016, Michifuri et al., 2012) included both neoplastic tissue and adjacent non-neoplastic tissue for analysis, allowing for comparison of markers in tumour and healthy tissue in the same patient. To this regard, Destro Rodrigues et al (2016) was the first to show that there was a dysregulation of CD44, Oct4, and Nanog in MEC tissue samples compared with non-neoplastic salivary gland tissue. Despite the limited sample size of this study (28 individuals), the information gathered from these authors can be used to supplement and drive further research in this area.

The study by Kim et al (Kim, Choung, Lee, Khwarg \& Kim, 2015) was the first to evaluate the expression of CSC-associated molecules as prognostic markers in eyelid sebaceous gland carcinoma. They were able to demonstrate that overexpressed markers, particularly ALDH1, can be used as predictors of lymph node metastasis. Habu et al (Habu et al., 2015) were the first to show that Oct3/4 were useful markers for prediction of distant neck metastasis development in OSCC lesions. Huang et al (Huang, Xu, Wu, Sun \& Zhang, 2014) was the first to examine the co-expression of CSC markers and reported that ALDH1 was correlated with Sox2 but not Oct4 and CD44 in OSCC lesions. Yang et al (Yang et al., 2013) revealed for the first time that cells with high expression of ALDH1 had CSC properties in Oesophageal squamous cell carcinoma (ESCC). Interestingly, a relatively large body of evidence now associates ALDH1 with metastasis, indicating that this marker could potentially be used as a predictive marker for metastasis during the diagnostic phase.

The use of CD133 as a molecular marker of cancer stemness is still controversial. Of the 13 articles evaluated, only 4 studies investigated CD133 as a possible CSC marker. Of these, only half (Kim, Choung, Lee, Khwarg \& Kim, 2015, Habu et al., 2015) were able to reach a conclusion in regard to CD133 being a possible CSC marker. Conversely, the other two studies (Mannelli et al., 2015, Tang et al., 2013) showed very weak or no link of CD133 to stemness. However, the former studies had larger number of participants (both 50 individuals), whereas the studies that did not

This article is protected by copyright. All rights reserved. 
show CD133 to be linked to CSCs had only 8 and 29 participants. Furthermore, all studies alluded to CD133 as only being present when metastasis had occurred, so perhaps the cohorts that did not count a large number of lymph node metastasis were not able to identify CD133. This suggests that CD133 may not be present in primary tumours but may be more prevalent in patients who have some degree of lymph node metastasis.

Although previous research focusing on Oct 4 did correlate this molecule with survival and prognosis, the authors in this systematic review concluded that more research is needed to understand the role of Oct4 in HNC. There were a handful of studies that demonstrated the presence of Oct4 in CSCs. Further, the importance of Oct4 in cancer progression was not confirmed in multiple studies. The main association of Oct4 was with MEC, where there was a strong link as well as a correlation associated with progression and worse prognosis. Thus, CSC-related Oct4 could play a role in a tumour or site-specific manner.

Interestingly, not a single putative CSC marker was identified in all studies, showing that there is variability amongst CSC markers between different malignancies. In addition, even in the same type of cancer, different markers were expressed showing that there is large variability of CSC markers in the same tumour. This review demonstrates that more research is needed in this area as it is a relatively new field of investigation. The identification of predictable and reliable CSC markers could be used as a tool for the selection of potential therapeutic agents as well as prognostic predictors in patients with HNC.

\section{Conclusion}

From this review, we conclude that there are some common CSC markers identified amongst many head and neck tumours, namely CD44, ALDH1, CD133, Nanog and Sox2. It is not yet known whether these markers are specifically associated with certain types of cancers. Furthermore, none of the molecules identified was expressed in all cancer samples in any study.

This suggests that a single common CSC sorting marker may not exist even within identical types of tumour. 


\section{Acknowledgements}

The authors would like to acknowledge the support of the Melbourne Dental School, The University of Melbourne.

\section{Funding statement}

None

\section{Conflict of interest}

The authors declare no conflict of interest.

\section{Author contribution}

SSY performed all steps of the systematic literature search, collected all data and wrote the first draft of the manuscript. NC generated the research ideas, overlooked the literature search, and wrote the final draft of the manuscript.

\section{References}

Adachi, K., Suemori, H., Yasuda, S., Nakatsuji, N., \& Kawase, E. (2010). Role of SOX2 in maintaining pluripotency of human embryonic stem cells. Genes To Cells. $15: 455-70$

Allegra, A., Alonci, A., Penna, G., Innao, V., Gerace, D., Rotondo, F., \& Musolino, C. (2014). The Cancer Stem Cell Hypothesis: A Guide to Potential Molecular Targets. Cancer Investigation, 32, 470-495.

Argiris, A., Karamouzis, M., Raben, D., \& Ferris, R. (2008). Head and neck cancer. The Lancet, 371, 1695-1709.

Boiani, M., \& Schöler, H. (2005). Regulatory networks in embryo-derived pluripotent stem cells. Nature Reviews Molecular Cell Biology, 6, 872-881.

Bourguignon, L., Wong, G., Earle, C., \& Chen, L. (2012). Hyaluronan-CD44v3 Interaction with Oct4-Sox2-Nanog Promotes miR-302 Expression Leading to Selfrenewal, Clonal Formation, and Cisplatin Resistance in Cancer Stem Cells from Head and Neck Squamous Cell Carcinoma. Journal Of Biological Chemistry, 287, 3280032824.

Chambers, I., Colby, D., Robertson, M., Nichols, J., Lee, S., Tweedie, S., \& Smith, A. (2003). Functional Expression Cloning of Nanog, a Pluripotency Sustaining Factor in Embryonic Stem Cells. Cell, 113, 643-655.

This article is protected by copyright. All rights reserved. 
Chang, C., Shieh, G., Wu, P., Lin, C., Shiau, A., \& Wu, C. (2008). Oct-3/4

Expression Reflects Tumor Progression and Regulates Motility of Bladder Cancer Cells. Cancer Research, 68, 6281-6291.

Clarke, M., Dick, J., Dirks, P., Eaves, C., Jamieson, C., \& Jones, D. et al. (2006). Cancer Stem Cells-Perspectives on Current Status and Future Directions: AACR Workshop on Cancer Stem Cells. Cancer Research, 66, 9339-9344.

de Jong, M., Pramana, J., van der Wal, J., Lacko, M., Peutz-Kootstra, C., \& de Jong, J. et al. (2010). CD44 Expression Predicts Local Recurrence after Radiotherapy in Larynx Cancer. Clinical Cancer Research, 16, 5329-5338.

Destro Rodrigues, M., Sedassari, B., Esteves, C., de Andrade, N., Altemani, A., de Sousa, S., \& Nunes, F. (2016). Embryonic stem cells markers Oct4 and Nanog correlate with perineural invasion in human salivary gland mucoepidermoid carcinoma. Journal Of Oral Pathology \& Medicine, 46, 112-120.

Faber, A., Barth, C., Hörmann, K., Kassner, S., Schultz, J.D., Sommer, U., SternStraeter, J., Thorn, C., \& Goessler, U.R. (2011). CD44 as a stem cell marker in head and neck squamous cell carcinoma. Oncology Reports. 26:321-326.

Garvalov, B., \& Acker, T. (2010). Cancer stem cells: a new framework for the design of tumor therapies. Journal Of Molecular Medicine, 89, 95-107.

Habu, N., Imanishi, Y., Kameyama, K., Shimoda, M., Tokumaru, Y., \& Sakamoto, K. et al. (2015). Expression of Oct3/4 and Nanog in the head and neck squamous carcinoma cells and its clinical implications for delayed neck metastasis in stage I/II oral tongue squamous cell carcinoma. BMC Cancer, 15, 730.

Horst, D., Scheel, S., Liebmann, S., Neumann, J., Maatz, S., Kirchner, T., \& Jung, A. (2009). The cancer stem cell marker CD133 has high prognostic impact but unknown functional relevance for the metastasis of human colon cancer. The Journal of Pathology, 219, 427-434.

Huang, C., Xu, X., Wu, T., Sun, Z., \& Zhang, W. (2014). Correlation of ALDH1, CD44, OCT4 and SOX2 in tongue squamous cell carcinoma and their association with disease progression and prognosis. Journal Of Oral Pathology \& Medicine, 43, 492-498.

Hur, M. (2009). Reviewing of cancer stem cell hypothesis. Korean Journal Of Clinical Oncology, 5, 20-24.

Ivanova, N., Dobrin, R., Lu, R., Kotenko, I., Levorse, J., \& DeCoste, C. et al. (2006). Dissecting self-renewal in stem cells with RNA interference. Nature, 442, 533-538.

Karatas, O., Suer, I., Yuceturk, B., Yilmaz, M., Oz, B., \& Guven, G. et al. (2016). Identification of microRNA profile specific to cancer stem-like cells directly isolated from human larynx cancer specimens. BMC Cancer, 16, 853.

This article is protected by copyright. All rights reserved. 
Kashyap, V., Rezende, N., Scotland, K., Shaffer, S., Persson, J., Gudas, L., \& Mongan, N. (2009). Regulation of Stem Cell Pluripotency and Differentiation Involves a Mutual Regulatory Circuit of the Nanog, OCT4, and SOX2 Pluripotency Transcription Factors With Polycomb Repressive Complexes and Stem Cell microRNAs. Stem Cells And Development, 18, 1093-1108.

Kim, N., Choung, H., Lee, M., Khwarg, S., \& Kim, J. (2015). Cancer Stem Cell Markers in Eyelid Sebaceous Gland Carcinoma: High Expression of ALDH1, CD133, and ABCG2 Correlates With Poor Prognosis. Investigative Opthalmology \& Visual Science, 56, 1813-1819.

Liberati, A., Altman, D.G., Tetzlaff, J., Mulrow, C., Gøtzsche, P.C., Ioannidis, J.P., Clarke, M., Devereaux, P.J., Kleijnen, J., \& Moher, D. (2009). The PRISMA statement for reporting systematic reviews and meta-analyses of studies that evaluate health care interventions: explanation and elaboration. Annals of Internal Medicine, 151, W65-94.

Linge, A., Löck, S., Gudziol, V., Nowak, A., Lohaus, F., \& von Neubeck, C. (2016). Low Cancer Stem Cell Marker Expression and Low Hypoxia Identify Good Prognosis Subgroups in HPV(-) HNSCC after Postoperative Radiochemotherapy: A Multicenter Study of the DKTK-ROG. Clinical Cancer Research, 22, 2639-2649.

Lu, C., Xu, F., Gu, J., Yuan, Y., Zhao, G., Yu, X., \& Ge, D. (2015). Clinical and biological significance of stem-like CD133 + CXCR4 + cells in esophageal squamous cell carcinoma. The Journal Of Thoracic And Cardiovascular Surgery, 150, 386-395.

Ma, I., \& Allan, A. (2010). The Role of Human Aldehyde Dehydrogenase in Normal and Cancer Stem Cells. Stem Cell Reviews And Reports, 7, 292-306.

Mannelli, G., Magnelli, L., Deganello, A., Busoni, M., Meccariello, G., Parrinello, G., \& Gallo, O. (2015). Detection of putative stem cell markers, CD44/CD133, in primary and lymph node metastases in head and neck squamous cell carcinomas. A preliminary immunohistochemical andin vitrostudy. Clinical Otolaryngology, 40, 312-320.

Michifuri, Y., Hirohashi, Y., Torigoe, T., Miyazaki, A., Kobayashi, J., \& Sasaki, T. et al. (2012). High expression of ALDH1 and SOX2 diffuse staining pattern of oral squamous cell carcinomas correlates to lymph node metastasis. Pathology International, 62, 684-689.

Morse, D., Psoter, W., Cleveland, D., Cohen, D., Mohit-Tabatabai, M., Kosis, D., \& Eisenberg, E. (2007). Smoking and drinking in relation to oral cancer and oral epithelial dysplasia. Cancer Causes \& Control, 18, 919-929

Murdoch, D. (2007). Standard, and novel cytotoxic and molecular-targeted, therapies for HNSCC: an evidence-based review. Current Opinion In Oncology, 19, 216-221

This article is protected by copyright. All rights reserved. 
Parkin, D., Bray, F., Ferlay, J., \& Pisani, P. (2005). Global Cancer Statistics, 2002. CA: A Cancer Journal For Clinicians, 55, 74-108.

Rahadiani, N., Ikeda, J., Makino, T., Tian, T., Qiu, Y., \& Mamat, S. et al. (2010). Tumorigenic Role of Podoplanin in Esophageal Squamous-Cell Carcinoma. Annals Of Surgical Oncology, 17, 1311-1323.

Saito, S., Onuma, Y., Ito, Y., Tateno, H., Toyoda, M., \& Hidenori, A. et al. (2011). Possible linkages between the inner and outer cellular states of human induced pluripotent stem cells. BMC Systems Biology, 5(Suppl 1), S17.

Seino, S., Shigeishi, H., Hashikata, M., Higashikawa, K., Tobiume, K., \& Uetsuki, R. et al. (2015). CD44high/ALDH1highhead and neck squamous cell carcinoma cells exhibit mesenchymal characteristics and GSK3 $\beta$-dependent cancer stem cell properties. Journal Of Oral Pathology \& Medicine, 45, 180-188.

Soave, D., Oliveira da Costa, J., da Silveira, G., Ianez, R., de Oliveira, L., Lourenço, S., \& Ribeiro-Silva, A. (2013). CD44/CD24 immunophenotypes on clinicopathologic features of salivary glands malignant neoplasms. Diagnostic Pathology, 8, 29.

Tang, K., Dai, Y., Tong, M., Chan, Y., Kwan, P., \& Fu, L. et al. (2013). A CD90+ Tumor-Initiating Cell Population with an Aggressive Signature and Metastatic Capacity in Esophageal Cancer. Cancer Research, 73, 2322-2332.

Vigneswaran, N., \& Williams, M. (2014). Epidemiologic Trends in Head and Neck Cancer and Aids in Diagnosis. Oral And Maxillofacial Surgery Clinics Of North America, 26, 123-141.

Yang, L., Ren, Y., Yu, X., Qian, F., Bian, B., \& Xiao, H. et al. (2013). ALDH1A1 defines invasive cancer stem-like cells and predicts poor prognosis in patients with esophageal squamous cell carcinoma. Modern Pathology, 27, 775-783.

This article is protected by copyright. All rights reserved. 


\section{Figures}

Figure 1. Flow chart of the selection process according to PRISMA guidelines.

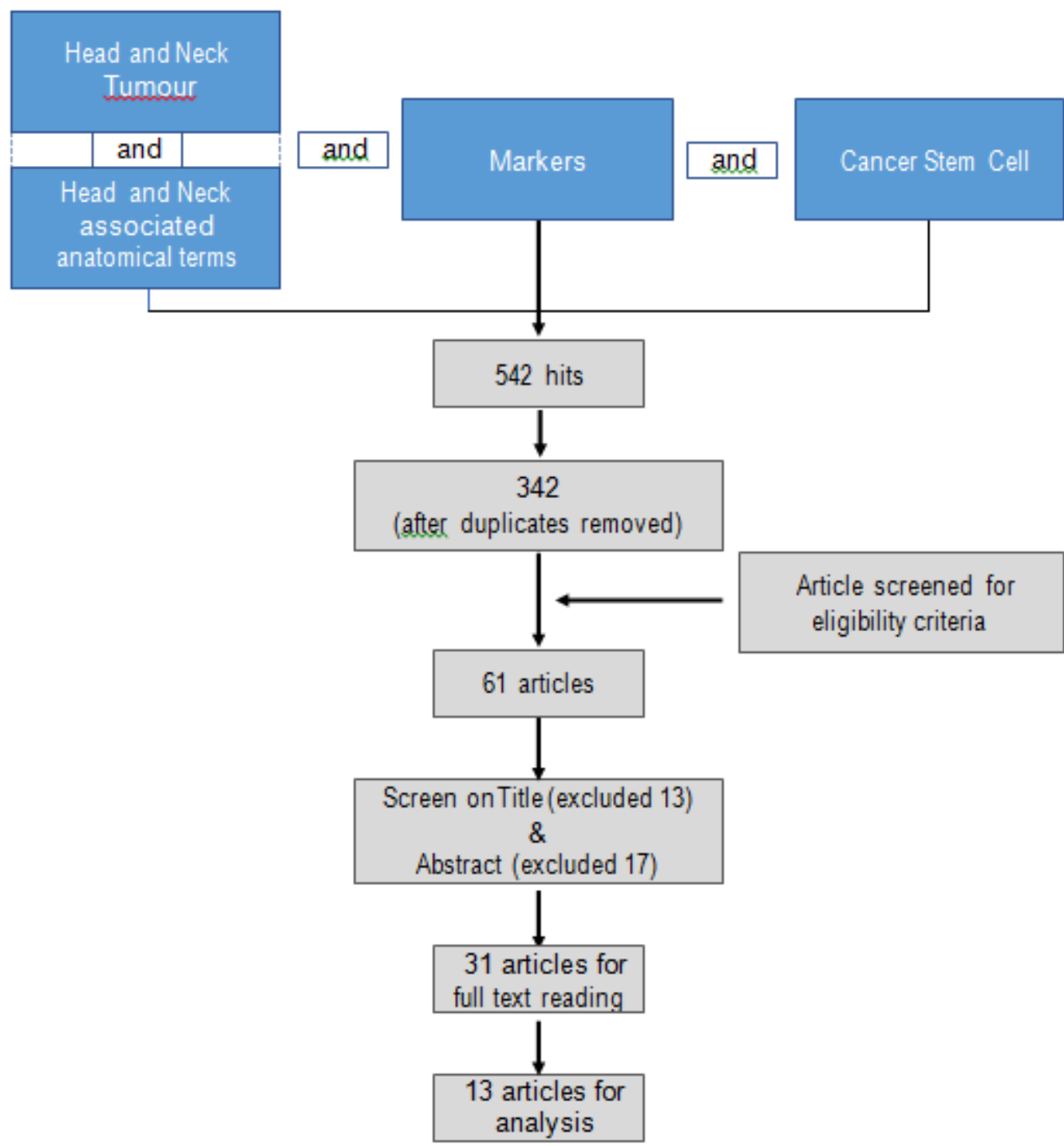

Figure 2. Schematic representation of the subcellular localization od CSC markers and their molecular function.
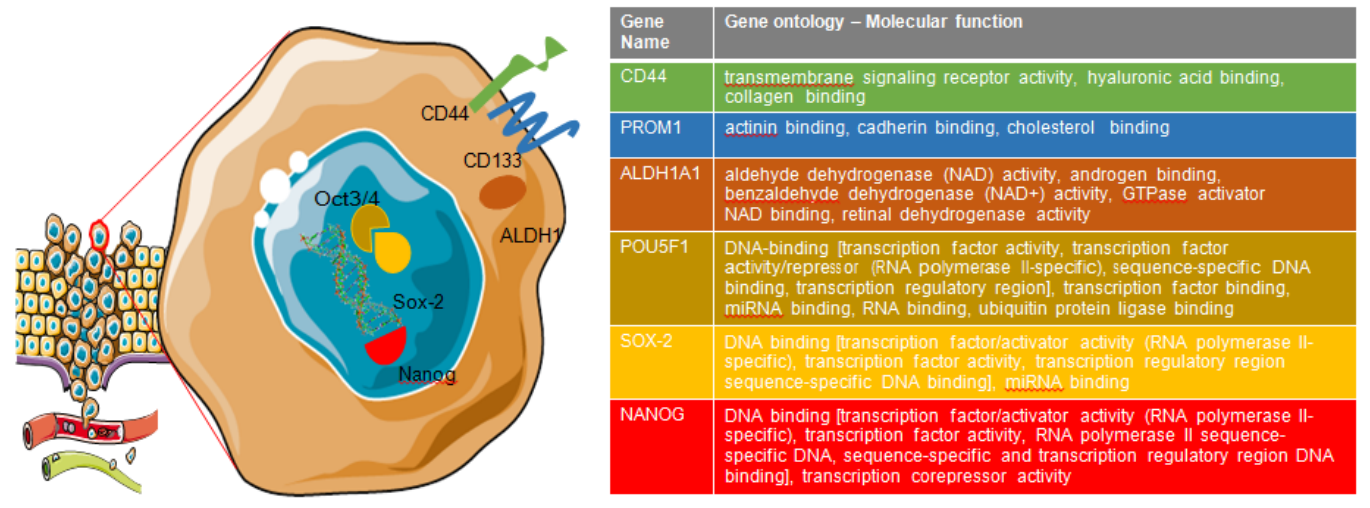

This article is protected by copyright. All rights reserved. 
Table I. Search terms used for this review

CATEGORIES

"HEAD AND NECK TUMO*”

“HeAd AND NecK” (ANatomical terms)

"Molecular MARKeR"

“CANCER STEM CELL"
ASSOCIATED TERMS

\author{
HEAD AND NECK AND (CANCER* OR \\ NEOPLASM* OR MALIGNAN* OR CARCINOMA* \\ OR SARCOMA
}
ORAL OR MOUTH OR NASAL OR SALIVARY GLAND* OR LARYNGEAL OR PHARYNGEAL OR OROPHARYNX OR SINUS OR MUCOSA OR FACE OR THROAT OR MANDIBLE OR MAXILLA
BIOMARKER OR IMMUNOHISTOCHEMISTRY OR PCR

NEOPLASTIC STEM CELL OR TUMO* STEM CELL

Table II. Overview of study characteristics of articles that were eligible for analysis

$\begin{array}{cccclc}\begin{array}{c}\text { Study } \\ \text { Author/s }\end{array} & \text { Year } & \begin{array}{c}\text { Sample } \\ \text { Size }\end{array} & \text { Cancer Type } & \begin{array}{l}\text { Molecular } \\ \text { marker/s }\end{array} & \text { Technique } \\ \text { Linge et al } & \mathbf{2 0 1 6} & \mathbf{1 9 5} & \text { HNSCC, } & \begin{array}{l}\text { CD44, MET, } \\ \text { SLC3A2, }\end{array} & \text { PCR }\end{array}$
Destro $2017 \quad 28 \quad$ Salivary Gland CD44, Bmi1, Rodrigues Mucopidermoid Oct4, Nanog et al Carcinoma,

IHC

$\begin{array}{ccccc}\text { Kim et al } 2015 & 50 & \begin{array}{c}\text { Eyelid } \\ \text { Sebaceous } \\ \text { gland } \\ \text { Carcinoma, }\end{array} & \begin{array}{l}\text { CD44, CD 133, } \\ \text { ALDH1, ABG2, } \\ \text { Oct4 }\end{array}\end{array}$

IHC

$\begin{array}{lllll}\text { Karatas et } & 2016 & 12 & \text { Larynx cancer } & \text { CD133, 0ct4 }\end{array}$ 


\begin{tabular}{|c|c|c|c|c|c|}
\hline Seino et al & 2016 & 3 & HNSCC & CD44, ALDh1, & RT-PCR \\
\hline Habu et al & 2015 & 50 & OSCC & $\begin{array}{l}\text { Oct3, Oct } 4 \text {, } \\
\text { Nanog }\end{array}$ & $\begin{array}{l}\text { RT-PCR, } \\
\text { IHC }\end{array}$ \\
\hline Lu et al & 2015 & 154 & Esophageal SCC & CD133, CXCR4 & IHC \\
\hline $\begin{array}{c}\text { Mannelli et } \\
\text { al }\end{array}$ & 2015 & 29 & HNSCC & CD44 & IHC \\
\hline $\begin{array}{c}\text { Huang et } \\
\text { al }\end{array}$ & 2014 & 66 & osCC (Tongue) & $\begin{array}{l}\text { CD44, ALDH1, } \\
\text { Oct4, Nanog, } \\
\text { Sox2 }\end{array}$ & IHC \\
\hline Yang et al & 2014 & 165 & Esophageal SCC & ALDH1A1 & IHC \\
\hline Tang et al & 2013 & 8 & Esophageal SCC & CD90+, CD44 & PCR \\
\hline $\begin{array}{l}\text { Michifuri } \\
\text { et al }\end{array}$ & 2012 & 80 & OSCC & ALDH1, Sox2 & IHC \\
\hline $\begin{array}{l}\text { Rahadiani } \\
\quad \text { et al }\end{array}$ & 2010 & 61 & Esophageal SCC & Podoplanin & IHC \\
\hline
\end{tabular}

Table III. Overview of markers associated with a statistically significant poorer prognosis. ( $\mathrm{P}<0.05$, unless otherwise specified)

\begin{tabular}{|c|c|c|c|c|}
\hline Markers & Prognostic significance & Study & $\begin{array}{l}\text { Cancer } \\
\text { Type }\end{array}$ & $\begin{array}{l}\text { Sample } \\
\text { Size }\end{array}$ \\
\hline $\begin{array}{l}\text { MET \& } \\
\text { SLC3a2 }\end{array}$ & $\begin{array}{l}\text { - Associated with } \\
\text { increased distant } \\
\text { metastases }\end{array}$ & $\begin{array}{c}\text { Linge et al, } \\
2016\end{array}$ & HNSCC & 195 \\
\hline
\end{tabular}




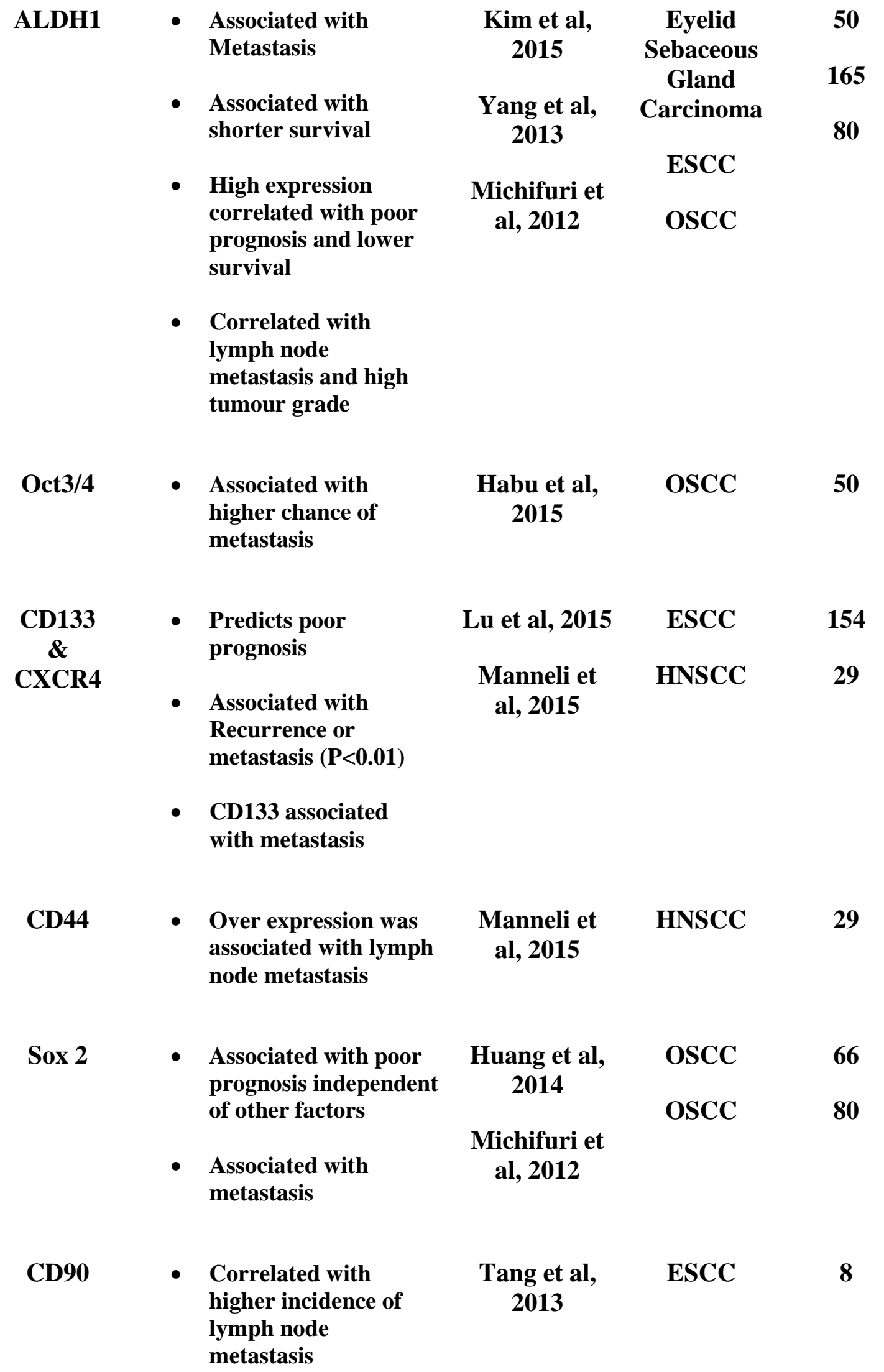




\section{University Library}

\section{- M M N E R VA A gateway to Melbourne's research publications}

Minerva Access is the Institutional Repository of The University of Melbourne

Author/s:

Yu, SS;Cirillo, N

Title:

The molecular markers of cancer stem cells in head and neck tumors

Date:

2020-01-01

Citation:

Yu, S. S. \& Cirillo, N. (2020). The molecular markers of cancer stem cells in head and neck tumors. JOURNAL OF CELLULAR PHYSIOLOGY, 235 (1), pp.65-73. https://doi.org/10.1002/ jcp.28963.

Persistent Link:

http://hdl.handle.net/11343/285996 